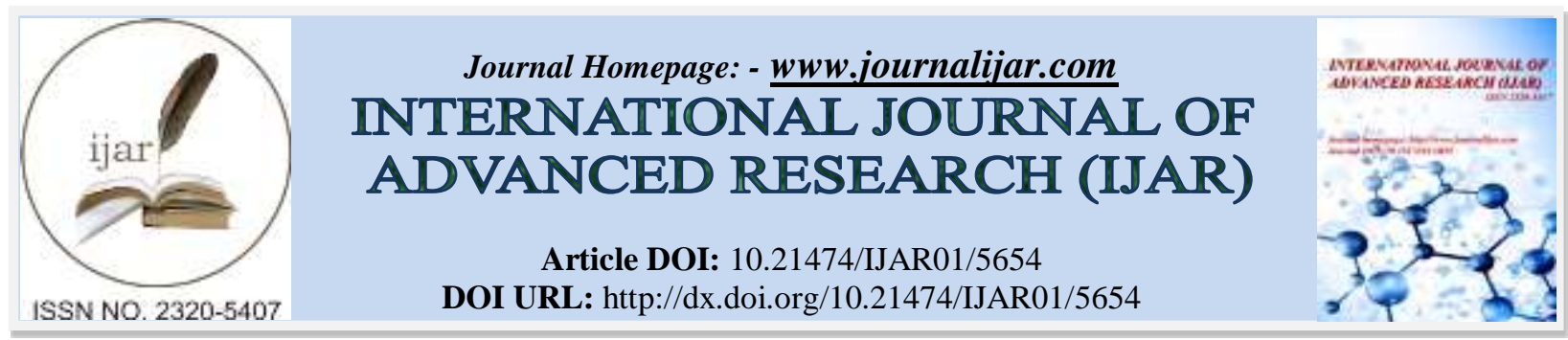

RESEARCH ARTICLE

\title{
INDIVIDUAL THEORY OF POVERTY AND INFORMAL SECTOR: LINKAGES OR ALTERNATIVE EXPLANATIONS.
}

Shweta Sharma.

Assistant professor, department of planning, school of planning and architecture, vijayawada, andhra pradesh, india.

\section{Manuscript Info}

Manuscript History

Received: 18 August 2017

Final Accepted: 20 September 2017

Published: October 2017

Key words:-

informal, Vijayawada, genetic factors, individualistic theory, poverty, age, education, period of stay.

\begin{abstract}
There has been a huge debate on the impact of individual factors, social factors and structural factors on earnings of people. This paper is an attempt to test the first set of factors, the individual factors in terms of their impact on earnings of street vendors in Vijayawada, Andhra Pradesh. 200 samples collected from two major markets of Vijayawada, One Town and Besant Road, assess the impact of genetic and personal reasons of vendors to be poor in the chosen case studies. The method of path co-efficient has been used to assess the relationships of each variable (age, education, period of stay) with incomes of vendors. The results depict that education has a strong positive relationship with incomes of a vendor. Period of stay is the second most important variable affecting earnings of vendors. It has a negative impact on incomes, i.e., more the duration of stay of vendors in their present occupation less are their chances to earn more. Age is the least important factor affecting income levels of vendors. However, these relationships undergo drastic change when two or more variable are taken together. The results have been tested on a class II city. However, the results might differ drastically if tested on a mega city such as Delhi, Mumbai, Chennai or Kolkata.
\end{abstract}

Copy Right, IJAR, 2017,. All rights reserved.

\section{Introduction:-}

Various theories have been given over the past explaining reasons for poverty. There are two prominent theories explaining existence of poverty in society; it is caused either caused by individual deficiency or it is caused by social set up. The latter theory can be further subdivided into various sub theories which pin down the explanation of poverty, first, on cultural belief systems supporting sub-cultures of poverty, second, on economic, political and social distortions or discrimination, third, on geographical disparities or, fourth, on cumulative and cyclical interdependencies.

This individual theory of poverty states that individuals are responsible for their poverty. Its proponents blame individuals for creating their own problems, and argue that with harder work and better choices the poor could have avoided (and now can remedy) their problems (Spencer 1979). Other variations of the individual theory of poverty ascribe poverty to lack of genetic qualities such as intelligence that are not so easily reversed. The second theory of poverty roots its cause in the "Culture of Poverty" (Lewis 1968). This theory suggests that poverty is created by the transmission over generations of a set of beliefs, values, and skills that are socially generated but individually held. Individuals are not necessarily to blame because they are victims of their dysfunctional subculture or culture. 
Whereas the first "individualistic" theory of poverty is advocated by conservative thinkers and the second is a culturally liberal approach, the third approach is a progressive social theory. Theorists in this tradition look not to the individual as a source of poverty, but to the economic, political, and social system which causes people to have limited opportunities and resources with which to achieve income and well-being. Much of the literature on poverty now suggests that the economic system is structured in such a way that poor people fall behind regardless of how competent they may be. Partly the problem is the fact that minimum wages do not allow single mothers or their families to be economically self-sufficient (Jencks 1996). The problem of the working poor is increasingly seen as a wage problem linked to structural barriers preventing poor families from getting better jobs, complicated by limited numbers of jobs near workers and lack of growth in sectors supporting lower skilled jobs (Tobin 1994).

Rural poverty, and poverty in developing countries is explained by a spatial characterization of poverty that exists separate from other theories. This theory states that people, institutions, and cultures in certain areas lack the objective resources needed to generate well-being and income, and that they lack the power to claim redistribution. The final theory of poverty looks at the individual and their community as caught in a spiral of opportunity and problems, and that once problems dominate they close other opportunities and create a cumulative set of problems that make any effective response nearly impossible (Bradshaw, 2000). The cyclical explanation explicitly looks at individual situations and community resources as mutually dependent, with a faltering economy, for example, creating individuals who lack resources to participate in the economy, which makes economic survival even harder for the community since people pay fewer taxes. This theory has its origins in economics in the work of Myrdal (1957) who developed a theory of "interlocking, circular, interdependence within a process of cumulative causation" that helps explain economic underdevelopment and development. Myrdal notes that personal and community wellbeing are closely linked in a cascade of negative consequences, and that closure of a factory or other crisis can lead to a cascade of personal and community problems including migration of people from a community. Thus the interdependence of factors creating poverty actually accelerates once a cycle of decline is started.

This paper explores the individual theory explanation for poor earnings of street vendors of Vijayawada. A main reason for low earnings of street vendors is explained by their lack of education and thus their lack of efforts to secure education which drove them to poverty. Genetic qualities such as intelligence are difficult to measure and thus have been excluded from the study. However age has been used as a proxy measure for hereditary attributes.

\section{Objective and Research Questions:-}

The objective of this paper is to examine whether the individual factors are responsible for the street vendors to be having less incomes. These individual factors can be genetic or lack of interest of vendors themselves to acquire education and skills to end up working in informal sector. The research questions are as follows:

a) What is the impact of age on income levels of vendors?

b) What is the impact of education on income levels of vendors?

c) What is the impact of PoS on the income levels of vendors?

\section{Case Area:-}

Vijayawada with a population of approximately 10.4 lakhs and spread over an area of $61.88 \mathrm{sq} \mathrm{km}$ is the most densely populated city of Andhra Pradesh state. It is one of the most rapidly growing urban markets of the country. Since the city has a high rate of migration from nearby towns and villages, thus it is not able to accommodate the migrant population in its formal sector. Thus, a major proportion of population is in the informal sector. The informal sector employs 17,176 people in the city which is $0.02 \%$ of the city population (MEPMA 2016). There are more than twenty-five locations in the city where the vendors occupy the space either as stationary or semistationary vendors. However, the city has twelve locations which have a predominant existence of vendors. Figure 1 shows the predominant vending locations in Vijayawada. 


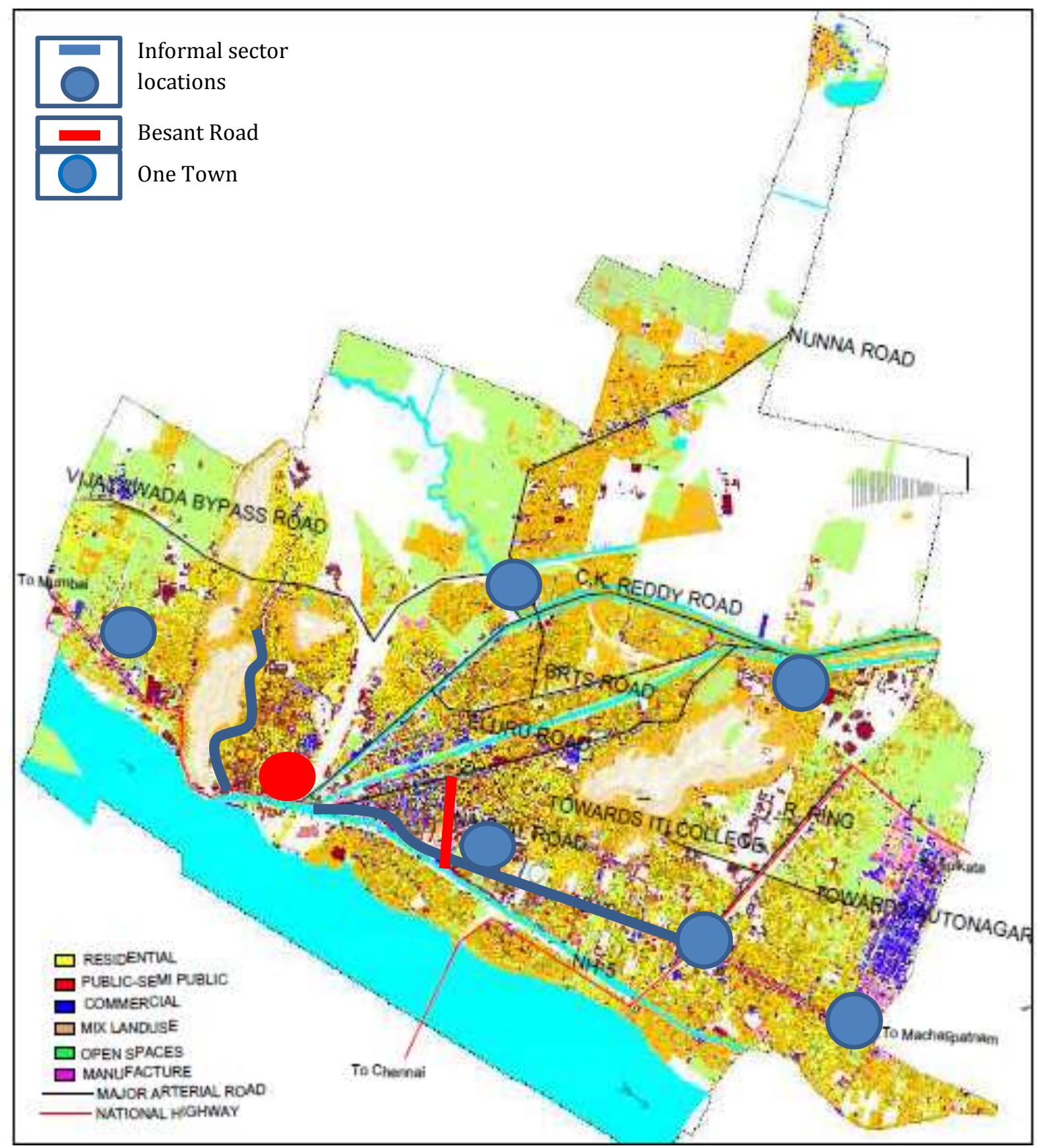

Figure 1:- Informal sector location in Vijayawada

Source: Authors' work

The study focusses on two major markets of the city, Besant Road and One town, which have a good concentration of vendors of the city. Although the city has good concentration of vendors in the city but only these markets have been chosen for study for the following reasons:

a) Both the markets have a good concentration of commercial shops which gives these markets a good enough clientele which is helpful for vendors in their business.

b) One Town is the oldest market of the city, which makes it essential to test parameters such as age and period of stay for the local as well as migrant dwellers of the city.

c) Besant Road in contrast is a new market which has arisen recently, yet attracts clientele because of the diversity of products available in the market and the ease of entry to this market place. 
d) All other locations for vendor concentration are random locations and not markets per se, which helps us in conducting the tests as market location is the controlling variable for both the chosen case areas. The results might differ is the control variable is not taken into consideration.

\section{Methodology:-}

\section{Participants}

The sample consisted of 200 street vendors located in two prominent markets of Vijayawada city: One town and Besant Road. The sample was selected with the method of purposive sampling where data was collected only for registered vendors in the chosen markets.

\section{Data collection and analysis}

The data was collected from primary and secondary sources. The data collected through secondary sources was the location map of vendors and their registration details. The primary data was collected through questionnaires. The closed and open ended questionnaire was chosen as substitute to interviews because semi-structured interviews would not help in getting in-depth understanding of the vendors. The closed ended questionnaire included questions about the socio-economic conditions of vendors. Likert scale was used to assess certain qualitative questions. In conclusion, the data was collected through both qualitative and quantitative methods to cross check the reliability and validity of results collected.

\section{Design and procedure}

The data was collected through the questionnaire in approximately two weeks. Post data collection from the field, path analysis was done to understand the impact of age and education on PoS and finally on income of street vendors. In this diagram (figure 1), age and education are considered to be exogenous variables, that is, their variance is assumed to be caused entirely by variables not in the causal model.

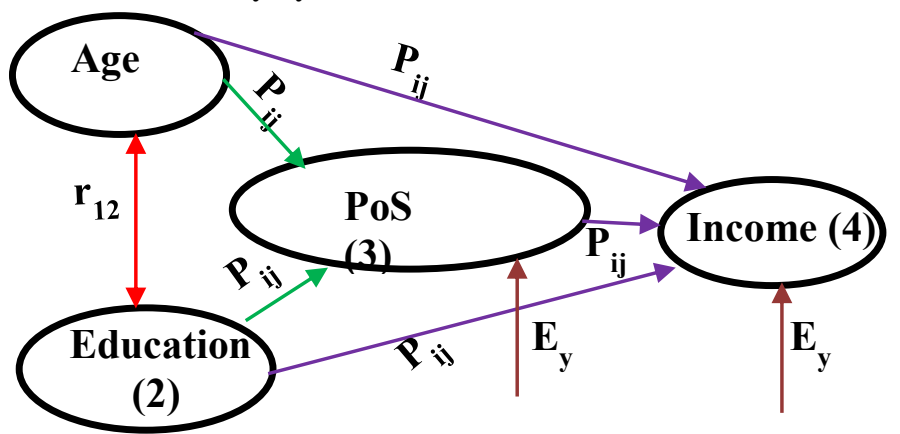

The connecting line with arrows at both ends indicates that the correlation between these two variables will remain unanalyzed because we choose not to identify one variable as a cause of the other variable. Any correlation between these variables may actually be casual ( 1 causing 2 and/or 2 causing 1) and/or may be due to 1 and 2 sharing common causes. For example, having a certain set of positive mentality may cause one to achieve education and may independently also have a strong relationship with his/ age, creating a spurious correlation between 1 and 2 that is totally due to their sharing a common cause, with no causal relationship between 1 and 2 . In this model, these relationships among the two factors have been deliberately ignored.

PoS and income are endogenous variables in this model - their variance is considered to be explained in part by other variables in the model. Paths drawn to endogenous variables are directional (arrowhead on one end only). Variance in PoS is theorized to result from variance in age, education and extraneous (not in the model) sources. The influence of these extraneous variables is indicated by the arrow from EY. Variance in income is theorized to be caused by variance in age, education, and extraneous sources.

For each path to an endogenous variable a path coefficient, pij, has been calculated, where "i" indicates the effect and " $\mathrm{j"}$ the cause. If we square a path coefficient we get the proportion of the affected variable's variable. The coefficient may be positive (increasing the causal variable causes increases in the dependent variable if all other causal variables are held constant) or negative (increasing causal variable decreases dependent variable). 


\section{Ethics}

The ethical principles of scientific research have been followed. The data of research were not distorted. The data was analyzed and interpreted, avoiding any possible bias and prejudices, respecting objectivity. As for the conduct of the research, the purpose of research was explained to protect the human subjects, respecting their autonomy and privacy.

\section{Results:-}

The values for age, education and PoS were normalised as they had different units, by the following formula: Normalised $X=\frac{\text { Actual Value-Minimum Value }}{\text { Maximum Value-Minimum Value }}$

Path analysis has been conducted as a hierarchical (sequential) multiple regression analysis. For each endogenous variable a multiple regression analysis was done predicting income (Y) from all other variables (age, education and place of stay) which are hypothesized to have direct effects on Y. We do not include in this multiple regression any variables which are hypothesized to affect Y only indirectly (through one or more intervening variables). The beta weights from these multiple regressions are the path coefficients shown in the typical figures that are used to display the results of a path analysis.

Path co-efficient model for Vijayawada markets is illustrated in Figure 2, to which path coefficients have been computed as below:

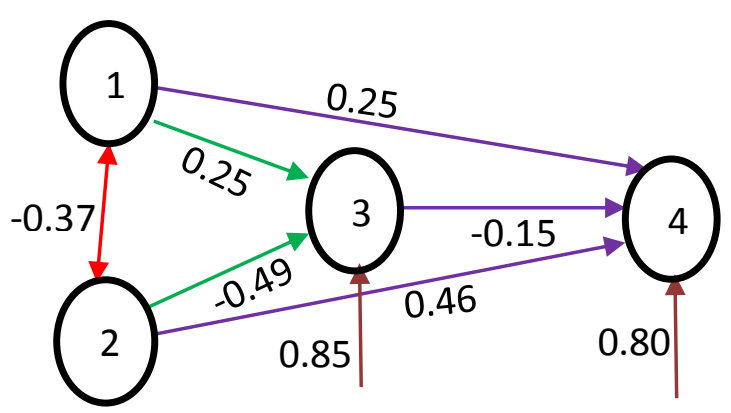

Path co-efficient for Age on PoS:

Direct: 0.25

Unanalysed due to education:

$-0.37 \mathrm{x}-0.49=0.18$

Total $=0.25+0.18=0.43$

Path co-efficient for Education on PoS:

Direct: -0.49

Unanalysed due to age:

$-0.37 \times 0.25=-0.09$

Total $=-0.49-0.09=-0.58$

Structural equation for PoS:

$0.43-0.58+0.85=0.7$

Path co-efficient for Age on Income:

Direct: 0.25

Indirect: $0.25 \mathrm{x}-0.15=-0.04$

Unanalysed due to education:

$-0.37 \times 0.46=-0.17$

Unanalysed due to education and PoS:

$-0.37 \mathrm{x}-0.49 \mathrm{x}-0.15=-0.03$

Total $=0.25-0.04-0.17-0.03=0.01$

Path co-efficient for Education on Income:

Direct: 0.46 
Indirect: $-0.49 \mathrm{x}-0.15=0.07$

Unanalysed due to age:

$-0.37 \times 0.25=-0.0925$

Unanalysed due to age and PoS:

$-0.37 \mathrm{x} 0.25 \mathrm{x}-0.15=0.014$

Total $=0.46+0.07-0.0925+0.014=0.45$

Path co-efficient for PoS on Income:

Direct: -0.15

Spurious:

PoS-Education-Income:

$-0.49 \times 0.46=-0.23$

PoS-Age-Income:

$0.25 \times 0.25=0.06$

PoS-Education-Age-Income:

$-0.49 \mathrm{x}-0.37 \mathrm{x} 0.25=0.083$

PoS-Age-Education-Income:

$0.25 \mathrm{x}-0.37 \mathrm{x} 0.46=-0.04$

Total: $-0.23+0.0625+0.083-0.04=-0.125$

Structural equation for income:

$0.01+0.45-0.125+0.80=\mathbf{1 . 1 4}$

Updated Path co-efficient model for Vijayawada markets is illustrated in Figure 2, to which path coefficients have been computed as below:

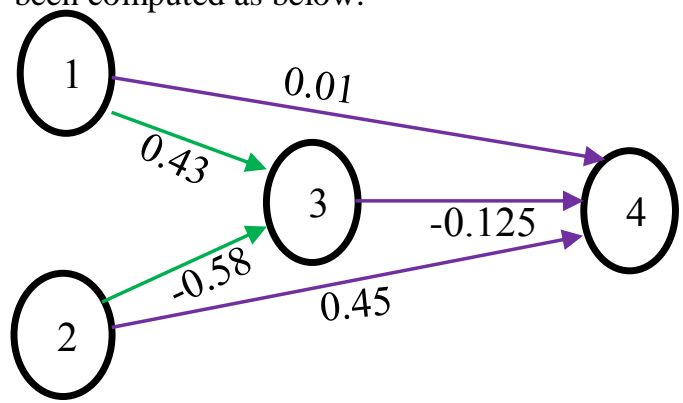

\section{Discussion:-}

The results depict that the overall path co-efficient for income is more than the path co-efficient for PoS. This means that the duration of length of hawkers in One Town and Besant Road is not that related to the genetic factors of age and education acquired. However, income seems to have a strong relationship with the age of the vendor, education acquired and PoS by the vendor in the chosen location.

Second, education has a stronger inverse bearing on the duration of stay in this occupation, i.e., more educated the vendor is less the probability of him to be in this profession. This is perhaps due to shift of vendors to formal sector after attainment of education and skill set. Age is positively related to the duration of stay of a vendor at a place. More the age of the person, more settled he becomes in his present occupation of vending.

Third, the education is more correlated to income levels of vendors than age or PoS. The more the educated the person, more are the chances for the vendors to earn. For examples, it was observed that if a person was aware of languages (English, Hindi) besides his local language (Telugu), he could attract more customers and thus earn more. However, PoS in this occupation has a negative bearing on income. More the PoS in this occupation lessons the chances of vendors to earn more. This implies it is better to shift to more formal sector over a period of time as vending becomes less remunerative over a period of time. Also, age has a positive relationship with income levels of vendors. The more aged the vendor, better his income was found to be.

Fourth, the spurious relationships between variables show the effect of sharing common variables on the regressed variable. The inverse relationship between PoS and income is strengthened if education acts as the spurious variable. 
This implies that lesser the period of time vendor spends in this occupation and lesser he is educated; more are his earnings. However, if the duration of stay in this occupation increases and the more educated the vendor becomes over a period of time, his chances to earn more in this occupation reduces because with better education and better understanding of the dynamics of the business, the person is more dissatisfied which adversely impacts his earnings. The relationship becomes positive between PoS and income if spurious variable is age. This implies that the age of the person becomes more dominant factor if seen in relationship with PoS and it influences the relationship of the $\mathrm{PoS}$ and income. Even though the duration of a vendor in his present occupation is less, yet more his age, will ultimately increase his earnings as a person would not like to shift from his occupation if he is old enough as it is his comfort zone, no matter he has been in this occupation for a very less time too.

When age and education are together spurious variables, the impact of PoS on income is varying. If the impact of age on PoS is ignored then it has a positive impact on income. This implies that if education of a person is more and his PoS in this occupation is also more, then both factors together have a negative impact on income levels of vendors. If impact of education on PoS is eliminated and only impact of age is considered, then more the age of the person and more the PoS in this occupation, more would be the income of the vendor.

Thus following relationships can be derived from the findings given above:

Impact of age on income levels of vendors

a) There is a positive relationship between age of a person and his income levels. More the age of the vendor, more are his earnings.

b) The age of a vendor is inversely related to his income if education and PoS are also considered. Better education and more duration of stay in this occupation reduce the income levels of vendors (immaterial of their age) due to their dissatisfaction in the job and urge to shift to more formal jobs.

Impact of education on income levels of vendors

a) Education has a positive and stronger relationship with income levels of vendors. More educated a vendor more are his chances to earn more. This is due to better communication skills and knowledge of various languages which makes it easier for vendors to attract customers and earn more money.

b) PoS and age have no impact on changing the relationship between education and income levels of vendors.

Impact of PoS on income levels of vendors

a) PoS has a negative relationship with income levels of vendors. More the period of stay in this occupation less his earnings, as there is a dissatisfaction and urge to move to more formal jobs and thus reduced interest in earning from informal sector.

b) PoS and education together further reduce the earnings of the vendor. This is because more education and more the duration of stay in this occupation further reduce the interest of vendors to be in informal sector and urges them to diversify and move towards formal sector.

c) PoS and age together improve the earnings of the vendors because more the age of the person better he is able to network and duration of stay in this occupation further helps him to develop better customer base which increases their earnings.

d) The same relationship implies between PoS and income if education and age are together considered as spurious variables.

\section{Conclusion:-}

The aim of undertaking this research was to examine whether the individual factors are responsible for the street vendors to be having less incomes. Through the path analysis method, it can be established that individual factors do have an impact on income levels of vendors. However, of all individual factors, lack of willingness to acquire education has a strong positive relationship with earnings of vendors. Genetic factors as age do favour more earnings of vendors, however, the relationship is not quite strong between the two variables. Period of stay in their present occupation has a negative bearing on income levels of vendors and is second important factor impacting earnings of vendors after education. This factor is also an individual trait of laziness where a person does want to diversify his earning base and move from informal to formal sector. This laziness of a vendor to stay in the same occupation for a long time, impact his earnings adversely. 
Through the research it becomes clear that genetic factors have less to do with low earnings of vendors. However, it is other individual factors of lack of motivation to acquire education and lack of motivation to move from formal to informal sector which adversely impact the earnings of vendors.

\section{Acknowledgements:-}

The author would like to acknowledge the street vendors who participated enthusiastically in the research and the Masters' students (at SPA Vijayawada), Anubhuti and Hema, who helped in data collection.

\section{References:-}

1. Bradshaw, T. K. (2000). Complex Community Development Projects: Collaboration, Comprehensive Programs and Community Coalitions in Complex Society. Community Development Journal, 35(2), 133-145.

2. Jencks, C. (1996). Can we replace welfare with work? in M. R. Darby (ed), Reducing Poverty in America (pp. 69-81). Thousand Oaks: Sage.

3. Lewis, 0. (1968). The Culture of Poverty. New York: Random House, Inc.

4. Mission for Elimination of Poverty in Municipal Areas (MEPMA). (2016). City-wise Street Vendors Report. Retrieved from http://www.apmepma.gov.in/streetVendorsCityReport.do

5. Myrdal, G. (1957). Economic Theory and Underdeveloped Regions. London: Gerald Duckworth and Co.

6. Spencer, H. (1979). Principles of Sociology, Vol.1, New York: D. Appleton.

7. Tobin, J. (1994). Poverty in Relation to Macroeconomic Trends, Cycles, Policies. in S. H. Danzinder, G. D. Sandefur, \& D. H. Weinberg (eds), Confronting Poverty: Prescriptions for Change . Cambridge: Harvard University Press. 